\title{
Study of thin, achromatic diffractive structures to focus terahertz radiation on a detector
}

\author{
Karolina LIEBERT $^{1 *}$, Martyna RACHON ${ }^{1}$, Andrzej KOlODZIEJCZYK ${ }^{1}$, Maciej SYPEK ${ }^{1}$, \\ IZABEla DUCIN $^{1}$, PrZemystaw ZAGRAJEK ${ }^{2}$, AgNiesZKa SIEMION ${ }^{1}$ \\ ${ }^{1}$ Warsaw University of Technology, Faculty of Physics, \\ 75 Koszykowa, Warsaw, Poland \\ ${ }^{2}$ Military University of Technology, Institute of Optoelectronics, \\ 2 Urbanowicz, Warsaw, Poland \\ ${ }^{*}$ Corresponding author: wegrzynska@if.pw.edu.pl
}

Thin and lightweight achromatic focusing elements with $F$-number close to 1 are desirable in many practical applications. We present the idea to use diffractive structures designed to work for the substantially increased $\mathrm{THz}$ frequency range. The paper analyses mono- and multi-focal lenses forming point-like foci as well as axicon and light sword optical elements focusing $\mathrm{THz}$ radiation into line segments located along the optical axis. We consider diffractive elements in a form of the first and the second order kinoforms having various thicknesses. Designed and fabricated elements were numerically and experimentally examined to verify their achromatic functioning. We present point spread functions ( $X Y$ scans) and $2 \mathrm{D}$ energy maps ( $X Z$ scans) for different $\mathrm{THz}$ frequencies. Moreover, a diagram of chromatic aberration is created by registering energy distribution along the optical axis for different frequencies. The distance corresponding to the highest energy is chosen for each frequency. Therefore, we can compare broadband working of designed structures. The spherical lens coded as kinoform of the second order provides the best broadband functioning, however it is two times thicker than structures providing extended depth of focus (light sword and axicon) working with slightly smaller efficiency but being much thinner.

Keywords: terahertz, diffractive optics, axicon, high order kinoform, spherical lens, light sword optical element, chromatic aberration.

\section{Introduction}

Large diversity of potential applications of terahertz $(\mathrm{THz})$ radiation caused growth of interest concentrated around this field $[\underline{1}, \underline{2}]$, nevertheless recently the main focus is on medicine [ $\underline{3}-\underline{6}]$, security [] $]$ or automotive industry [ $\underline{8}]$. These cases require not only efficient focusing of $\mathrm{THz}$ radiation, but mostly also the ability of broadband working. Better results could be obtained when THz systems could work at two or more different frequencies enabling spectral differentiation of examined objects. Thus, the develop- 
ment of optical components should take into account achromatic solutions allowing to work at many different frequencies and assuming real applications, additionally functioning in so-called atmospheric windows [9]

A common choice for broadband shaping of THz waves are metallic mirrors. Their main advantage is that they are free from chromatic aberration, which makes them wavelength independent. However, they are difficult to manipulate and very sensitive to any misalignments. Additionally, they require a lot of space and mostly are expensive $[\underline{10}, \underline{11}]$.

The next possibility to focus broadband $\mathrm{THz}$ beam is to use refractive or diffractive lenses. They are much easier in positioning in comparison to their mirrored counterparts. Refractive lenses in relation to diffractive ones are thicker, heavier and require more material that additionally absorbs radiation. Using diffractive elements enables designing lenses with $F$-number smaller or equal to 1 that can be relatively easily manufactured, in many cases using 3D printers. Additionally, in such a case, a distance between the thin diffractive element and its back focal spot coincides with the designed focal length, what in many cases is advantageous. Moreover, the possibilities given by different advanced diffractive elements for $\mathrm{THz}$ range are up to date for diverse applications, due to large versatility of possible manufacturing techniques and accessible materials [12]. Thin diffractive optical elements unfortunately have large chromatic aberration - resulting from designing them as structures introducing particular phase retardation, which is strongly dependent on the design wavelength (DWL). However, reducing of chromatic aberration can be realized by structures called kinoforms of higher order [13] - introducing phase shift with an almost continuous phase profile with a maximal phase value being a multiplicity of $2 \pi$. The more broadband working we assume, the thicker the designed element should be, and then we meet the condition in which an optical element becomes thick and therefore attenuates a lot of radiation.

For this reason, we analyze the possibility of using thin $\mathrm{THz}$ structures in a broad range of frequencies. Thus, we discuss different types of optical structures being a kinoform of small order (first or second) to assure focusing of terahertz radiation from some range of frequencies. These structures are: the spherical focusing lens, the multi-focal lens, the axicon and the light sword optical element. The preliminary results describing focusing properties of spherical lenses, axicons and light sword elements were already presented [14].

The aim of this study is to examine broadband possibilities of focusing of terahertz radiation on the detector - here, we assumed the range between 150 and $600 \mathrm{GHz}$. The limits were defined taking into account the fact that above $600 \mathrm{GHz}$ the water vapor present in the air highly absorbs the radiation and below $150 \mathrm{GHz}$ low spatial resolution is observed due to longer wavelengths (the same problem exists for millimeters waves) [15]. To verify broadband working of designed elements, we used three frequencies that were experimentally analyzed: $150 \mathrm{GHz}, 300 \mathrm{GHz}$ (corresponding to $1 \mathrm{~mm}$ chosen as a DL) and $600 \mathrm{GHz}$, and all of them are close to atmospheric windows [9]. 


\section{Designed elements}

In this paper, we characterize five different elements designed for broadband working as thin diffractive structures. Four of them are analyzed as achromatic THz optical elements, while one is used as a reference structure. A spherical lens coded as the first order kinoform (SLH1) enables comparison of designed elements with already known solution that suffers from large chromatic aberration. As it was already mentioned, high order kinoform structures are well known approach to suppress chromatic aberration in diffractive elements [16]. In many cases manufacturing thin and lightweight elements is advantageous, thus only one structure being the second order kinoform is considered in a form of the spherical lens (SLH2). Another method of reducing chromatic aberrations is based on using multi-focal lenses (MFLs) [17] or structures providing the increased depth of focus, like: light sword (LS) optical elements [18] or axicons (AX) [19]. The MFL discussed in this paper is composed of three equal areas (central circular and two annular) corresponding to three different lenses designed for three different wavelengths. Therefore, theoretically it exhibits achromatic behavior for these wavelengths. The axicon (AX) focuses incoming plane wave in a line segment located along the optical axis. It is a radially modulated structure. The light sword (LS) element has angular modulation and focuses radiation in the spiral segment stretched along and around the optical axis (very close to it). Together with extending the focal spot into a line segment it is possible to increase the chances of focusing the radiation with different frequencies at a determined distance. In other words, the increased depth of focus may result in focusing all considered wavelengths (or part of them) in a similar way into some overlapping line-segments along the optical axis. Thus, the chromatic aberration can be reduced as we are able to find such locus where all desired frequencies form a focal spot. Energy of the radiation focused at a particular distance is lower than for the reference structure SLH1 due to the fact that it is distributed along an elongated focal curve.

Table 1 presents all designed structures, analyzed numerically and experimentally in the paper. The modelled elements had a diameter of $50 \mathrm{~mm}$ and high numerical apertures, thus it was necessary to use a non-paraxial designing approach [20]. All formulas describing phase distributions are given in Table 1. Here, $f$ is the designed focal length equal to $50 \mathrm{~mm}, \lambda$ is the DWL. In a case of the axicon - parameters used in simulations were as follows: $a=\left(d_{2}-d_{1}\right) / R^{2}$ ( $R$ is the radius of the axicon aperture), $d_{2}=90 \mathrm{~mm}, d_{1}=20 \mathrm{~mm}$ and $g=2 a d_{2}$ [19], where $d_{1}, d_{2}$ define positions of the beginning and the end of the focal line segment behind the axicon. For the light sword element: $s=f_{1}+\left(f_{2} \Theta\right) / 2 \pi, f_{1}=40 \mathrm{~mm}$ and $f_{2}=80 \mathrm{~mm}$, where $f_{1}$ and $f_{2}$ determine distances between the structure and endpoints of the focal segment [21]. In a case of the MFL, the parameter $\lambda_{1,2,3}$ denotes DWL for each of three areas and is equal to $2 \mathrm{~mm}$ (central circular area), $1 \mathrm{~mm}$ (inner annular area) and $0.5 \mathrm{~mm}$ (external annular area), respectively. 
$\mathrm{T} \mathrm{a} \mathrm{b} \mathrm{l} \mathrm{e} \mathrm{1.} \mathrm{Designed} \mathrm{diffractive} \mathrm{elements.} \mathrm{Type} \mathrm{of} \mathrm{the} \mathrm{element;} \mathrm{phase} \mathrm{distribution} \mathrm{of} \mathrm{designed} \mathrm{optical}$ elements corresponding to the depth map, where black introduces 0 and white $2 \pi$ phase shift ( $4 \pi$ for SLH2); photographs of manufactured elements; formulas describing phase distribution of the designed optical elements.

\begin{tabular}{|c|c|c|c|}
\hline $\begin{array}{l}\text { Type of } \\
\text { element }\end{array}$ & $\begin{array}{l}\text { Phase } \\
\text { distribution }\end{array}$ & $\begin{array}{l}\text { Photograph } \\
\text { of elements }\end{array}$ & Formula \\
\hline SLH1 & & & $-\frac{2 \pi}{\lambda} \sqrt{r^{2}+f^{2}}$ \\
\hline SLH2 & & & $-\frac{2 \pi}{2} \sqrt{r^{2}+f^{2}}$ \\
\hline $\mathrm{AX}$ & & & $-\frac{\pi}{\lambda a} \ln \left|2 a \sqrt{a^{2} r^{4}+(1-g) r^{2}+d_{2}^{2}}+2 a^{2} r^{2}-g+1\right|$ \\
\hline LS & & & $-\frac{2 \pi}{\lambda} \sqrt{r^{2}+s^{2}}+\frac{2 \pi}{\lambda} s$ \\
\hline MFL & & & $-\frac{2 \pi}{\lambda_{1,2,3}} \sqrt{r^{2}+f^{2}}$ \\
\hline
\end{tabular}

Parameters of LS and AX elements were chosen and optimized to obtain the smallest focal spot at a constant and specified distance behind the structure assuming broadband illumination. To obtain the smallest point spread function (PSF) averaged for different frequencies, its Fourier transform was calculated and the broadest one was chosen.

\section{Computer modelling}

Computer modelling for all structures was carried out using Light Sword 6.0 software which is available in Laboratory of Optical Information processing at Faculty of Physics at Warsaw University of Technology (Poland). The propagation algorithm uses the modified convolution approach. Theoretically, in wave approach it is assumed that op- 
tical structure is represented by amplitude and phase distributions placed in one plane. In reality all elements have particular thickness, which was taken into account in the calculation. Thus, propagation through a structure was calculated using the modified convolution approach and the modified beam propagation method [22]. Due to the $F$-number $\leq 1$ an off-axis approach was applied [23].

Numerical simulations were carried out on calculation arrays of $4096 \times 4096$ pixels with the sampling $117 \mu \mathrm{m} \times 117 \mu \mathrm{m}$ (which was determined by the resolution of 3D printing machine, used for manufacturing of the elements). Calculated phase distributions of all optical elements are presented in Table 1.

In this paper we compare designed structures by analyzing three values: size of PSFs, focusing range in $X Z$ scans $(\mathrm{EnM})$ and inclination of the slope in chromatic aberration diagrams. At first, an energy distribution was registered in the focal plane of the studied structure. The energy is proportional to the intensity distribution used in numerical simulations and to power detected in the experiment, thus for clarity we everywhere use energy distribution. Such $X Y$ scans were performed for all structures at the same distance. Assuming illumination with a divergent beam coming from the source located $z_{\mathrm{e}}=1 \mathrm{~m}$ before the structure, the scan of the $X Y$ surface $(30 \mathrm{~mm} \times 30 \mathrm{~mm})$ was performed at a distance $z_{\mathrm{d}}=53 \mathrm{~mm}$. According to the equation $1 / z_{\mathrm{d}}=1 / \mathrm{f}-1 / z_{\mathrm{e}}$ this distance indicates the focal plane of the SLH1 illuminated by the above divergent beam. Registered distributions correspond to PSFs of the elements.

Then, energy patterns along propagation axis were scanned in $X Z$ plane. They correspond to EnM distributions. Here, we also take into account the fact that we use the same illumination of the structures and their positions, thus $X Z$ scans were carried out

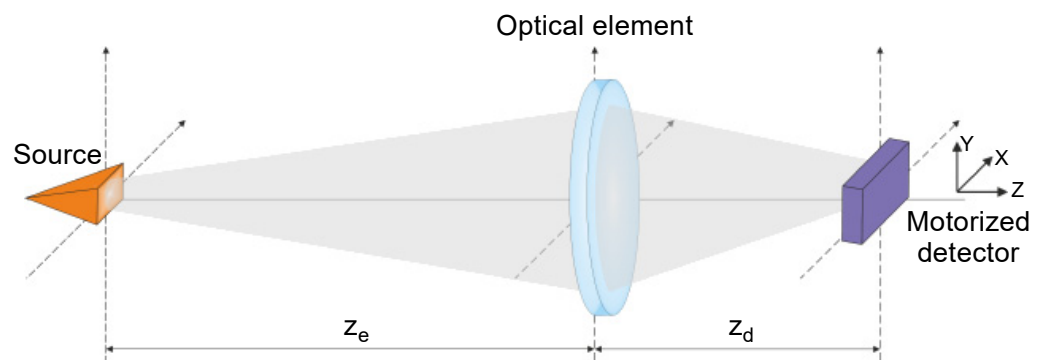

a



b

Fig. 1. The scheme of the optical setup (a) and a photograph of the real experimental setup (b) used to measure point spread functions (PSFs) and energy maps of the examined elements. The distance $z_{\mathrm{d}}$ depended on the method. For energy maps it was in the range from $35 \mathrm{~mm} \times 85 \mathrm{~mm}$, for PSF it was equal to $53 \mathrm{~mm}$, while to create a diagram of chromatic aberration, it varied from $20 \mathrm{~mm}$ to $100 \mathrm{~mm}$. 
having the size $36 \mathrm{~mm} \times 50 \mathrm{~mm}$ with $z_{\mathrm{d}}=35 \mathrm{~mm}$ to $z_{\mathrm{d}}=85 \mathrm{~mm}$. Chromatic aberration diagrams are described later in Section 6.

The scheme of the optical setup used for simulations and experimental evaluation is illustrated in Fig. 1a, additionally a photograph of the real setup with marked beam paths is shown in Fig. 1b.

Moreover, PSF and EnM were also calculated for $0.5 \mathrm{~mm}(600 \mathrm{GHz})$ and $2 \mathrm{~mm}$ $(150 \mathrm{GHz})$ which correspond to the boundary values of the assumed frequency range. Table 2 shows the results of numerical simulations carried out for 5 structures - SLH1, SLH2, AX, LS and MFL.

$\mathrm{T}$ a b 1 e 2. Computer modelling results of energy maps and point spread functions for three different frequencies: 150,300 , and $600 \mathrm{GHz}$ registered as $X Y$ scans at distance $z_{\mathrm{d}}=53 \mathrm{~mm}$ from the optical structure and $X Z$ scans in $35 \mathrm{~mm}$ to $85 \mathrm{~mm}$ range.

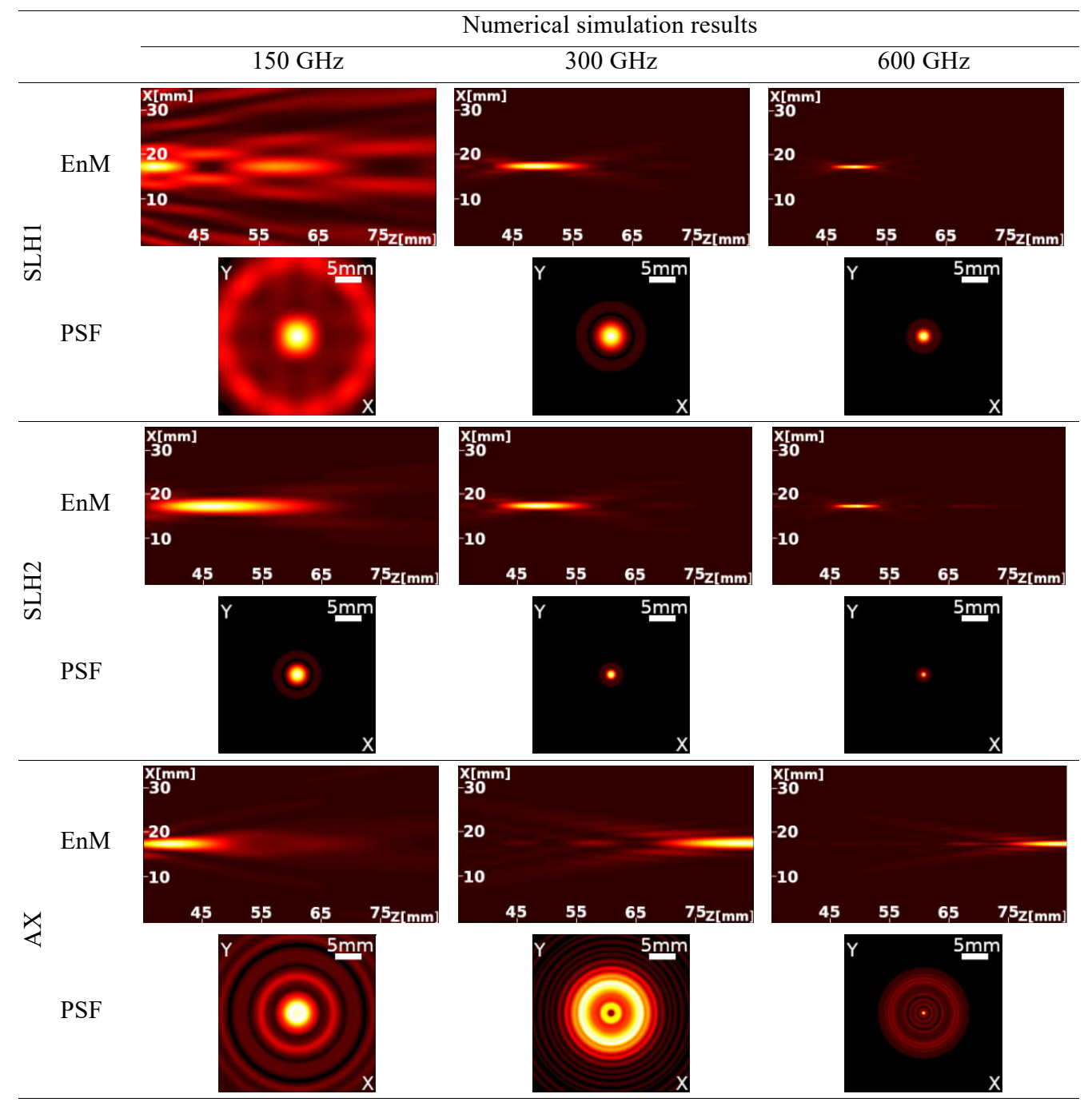


Ta b l e 2. Continued.

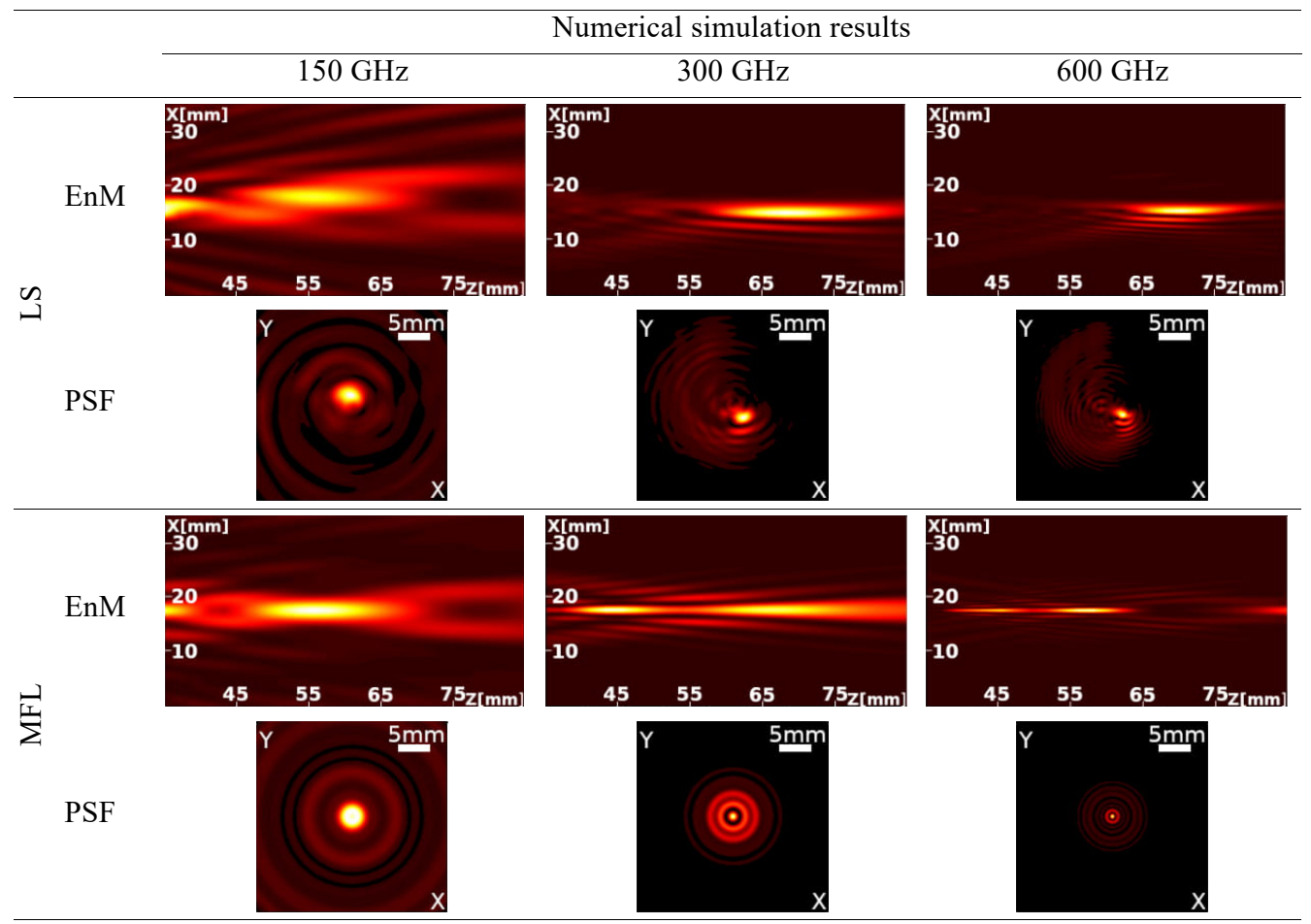

Elements SLH1, AX, LS and MFL are the first order kinoforms for the design frequency $300 \mathrm{GHz}$ and thus they are the second order kinoforms for the frequency $600 \mathrm{GHz}$. Therefore, they are focusing radiation at the same distance for both frequencies. Element SLH2 is the second order kinoform for a frequency $300 \mathrm{GHz}$, and thus it is the first and the fourth order kinoform for 150 and $600 \mathrm{GHz}$, respectively. Therefore, SLH2 element should focus the radiation for all three frequencies at the same distance behind the structure. Obviously, the spot size is proportional to a wavelength, therefore it is larger for longer wavelengths (smaller frequencies).

\section{Manufacturing of elements}

All optical elements were manufactured using the selective laser sintering (SLS) as 3D printing method [24] using polyamide 12 (PA12) material [25]. This material is easy in manufacturing and gives the possibility to obtain small details of structures with proper resolution. Designed structures had the diameter equal to $50 \mathrm{~mm}$ and some of them were not axially symmetrical elements. That is why a SLS 3D printing was chosen for manufacturing.

Photographs of manufactured structures are shown in Table 1. The refractive index $n$ of PA12 for all frequencies is equal to approximately 1.59 , which results in the maximal thickness of the structure equal to $1.69 \mathrm{~mm}$ for SLH1, AX, LS and MFL and 
$3.38 \mathrm{~mm}$ for SLH2. Maximal thickness of the structure $\left(h_{\max }\right)$ will introduce different maximal values of phase shift $\Phi$ for wavelengths other than DWL according to the equation: $\Phi_{\max }=\left(2 \pi h_{\max }(n-1)\right) / \lambda$.

Uniform value of refractive index over a chosen frequency range results in no dispersion. However, the absorption coefficient is strongly dependent on the frequency and is equal to: $0.5 \mathrm{~cm}^{-1}(150 \mathrm{GHz}), 1 \mathrm{~cm}^{-1}(300 \mathrm{GHz})$ and $7 \mathrm{~cm}^{-1}(600 \mathrm{GHz})$. Such values of absorption coefficient introduce significant attenuation losses inside the structures, especially for higher frequencies. Moreover, the source of $\mathrm{THz}$ radiation used in the experiment has smaller maximal power for higher frequencies and thus the quantitative comparison of results could be misleading in this case. For this reason only qualitative verification is performed.

\section{Experimental evaluation}

The measurements were done with a frequency multiplier from VDI (Virginia Diodes, Inc., Charlottesville, VA, USA). The examined optical elements (OE) were illuminated with a divergent beam. The distance between the source and the examined structure coincided with the performed simulations and was equal to $z_{\mathrm{e}}=1 \mathrm{~m}$. Then, $X Y Z$ scans were created using a VDI detector mounted on the motorized stages. The experimental optical system is presented in Fig. 1. All emitters were forming divergent beams with the divergence angle between 11 and 13 degrees. Detectors were also equipped with horn antennas with aperture dimensions from 2.4 to $8.4 \mathrm{~mm}$ (depending on the scanning frequency). The size of the horn for the emitter and the detector for particular frequency was the same. To measure the signal from the detector, a lock-in system (Stanford Research Systems SR830) based on modulation at $187 \mathrm{~Hz}$ was used.

EnMs were scanned with the resolution of $2 \mathrm{~mm}$, while PSFs were registered with a scanning step equal to $1 \mathrm{~mm}$. Distances $z_{\mathrm{e}}$ and $z_{\mathrm{d}}$ were measured from the examined structure to the middle of the length of the horn.

Focal spots and focal line segments are compared in the form of energy maps and point spread functions in simulations (Table 2) and experiments (Table 3 ). It is clearly seen that spherical lens being a kinoform of the first order (SLH1) focuses incident radiation at a distance of $53 \mathrm{~mm}$ for both $300 \mathrm{GHz}$ (DWL) and $600 \mathrm{GHz}$. For the latter frequency such structure works as a kinoform of the second order, thus the clear focal spot is also observed at a desired distance. In case of illumination with light having smaller frequency, a created energy pattern consists of primarily noise. Spherical lens coded as the second order kinoform (SLH2) forms the focal spot at a desired distance for all three analyzed frequencies. It should be underlined that the sizes of the focal spots for spherical lenses vary depending on the wavelength of an illuminating beam. Together with an increased frequency one can observe smaller focal spot which perfectly coincides with the performed experiments.

To get a better achromatic effect, the parameters of structures focusing light into line segments (AX and LS) were intentionally optimized for broadband THz radiation. For monochromatic $\mathrm{THz}$ beams the concentration distances are generally different. In 
particular cases of frequencies $300 \mathrm{GHz}$ and $600 \mathrm{GHz}$ they are larger than $53 \mathrm{~mm}$ which is illustrated in Tables 2 and 3. It is worth noticing that in case of LS element the distribution in a focal plane has the shape of a small spot with characteristic tail - rotating around the optical axis from $0^{\circ}$ to $360^{\circ}$ within the assumed line-segment. Multi-focal lens (MFL) also focuses the radiation further than $53 \mathrm{~mm}$ (like AX and LS) and for all three frequencies we can find a focal plane around $60 \mathrm{~mm}$. The energy distributions in the experimental part have smaller resolution due to the size of the detector. Nevertheless, in applications the real size of the horn antenna must be considered, it results in larger sampling in experiment than in simulations. Relatively large size of the horn

$\mathrm{T}$ a b $1 \mathrm{e}$ 3. Experimental results of energy maps registered in $X Z$ plane and point spread functions registered as $X Y$ scans at a distance $z_{\mathrm{d}}=53 \mathrm{~mm}$ from the optical structure.

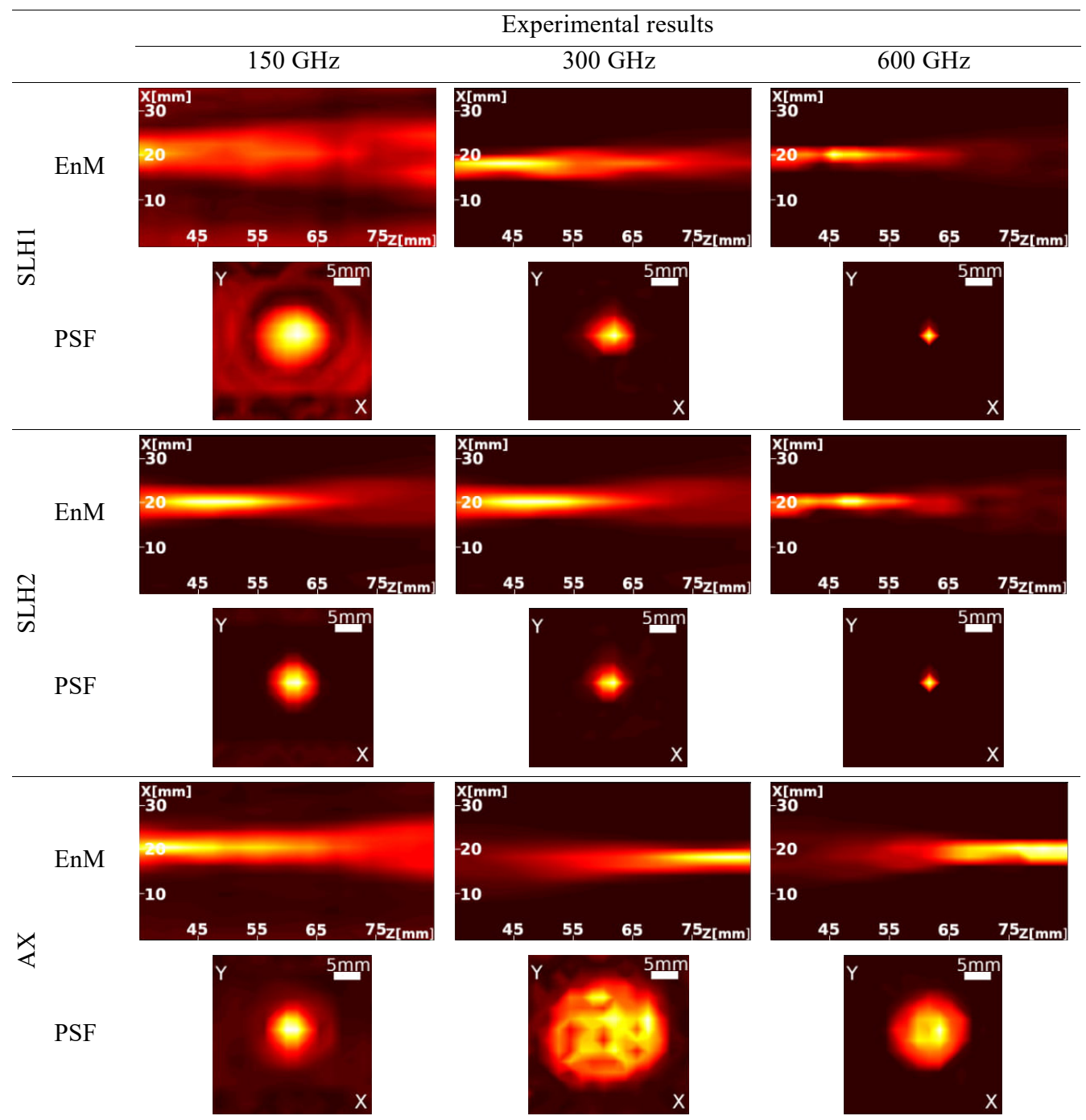


T a b l e 3. Continued.

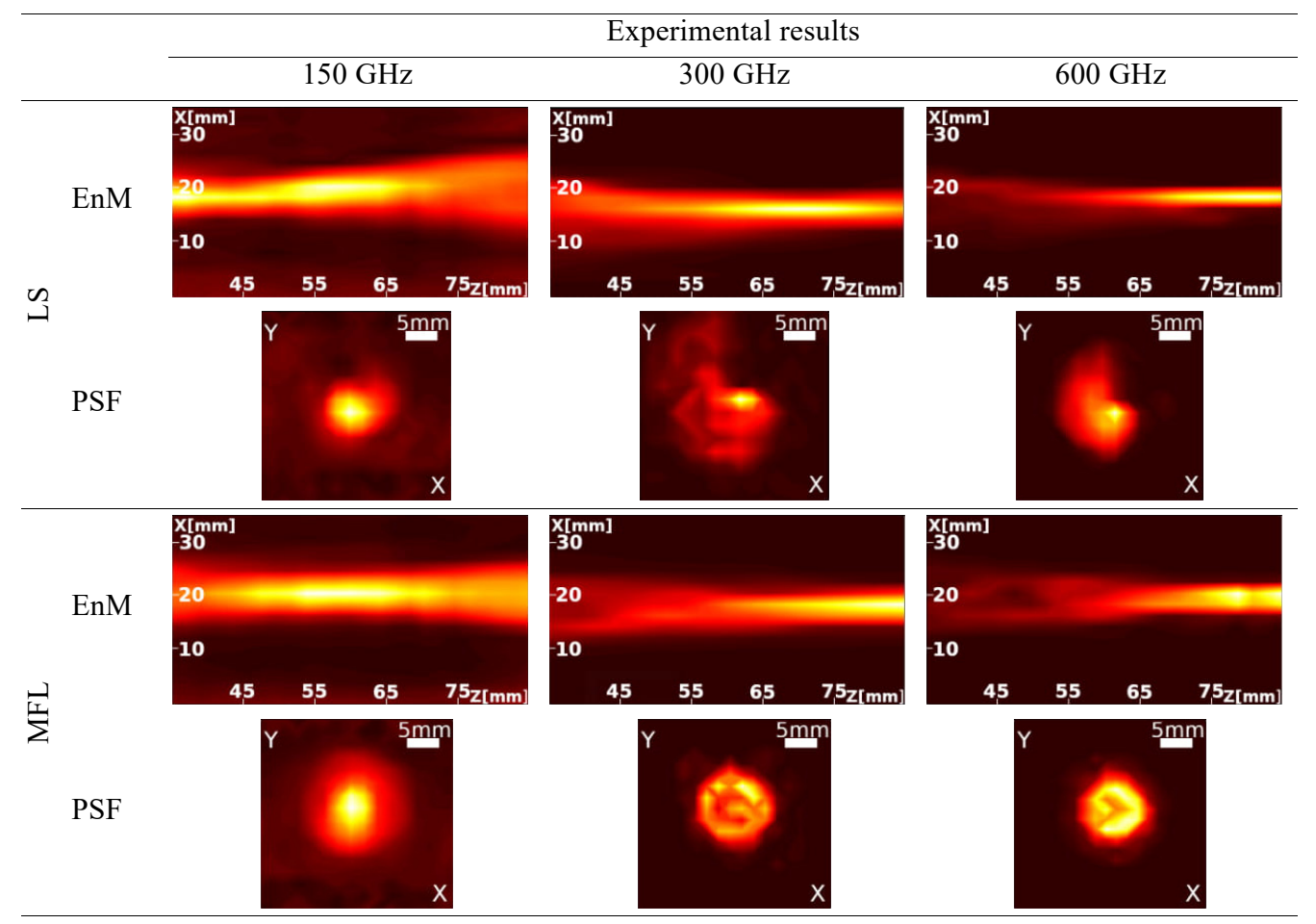

antenna (few millimeters) in comparison to pixel size (hundreds of micrometers) enables to collect energy propagating along the optical axis and a little bit around which assures better broadband properties. Even though some frequencies have larger spot sizes - they will be still registered by the detector.

\section{Chromatic properties}

Apart from the results given in Tables 2 and 3, the chromatic properties of the studied elements (SL, MFL, LS and AX) can be analyzed quantitatively by the focal spot shift $z_{f_{\max }}$ depending on a frequency. This shift is calculated in respect to a position of a focal spot for a reference frequency $300 \mathrm{GHz}$. The dependence of $z_{f_{\max }}$ on frequency for all elements forms a chromatic aberration diagram shown in Fig. 2. The red line $z_{f_{\max }}=0$ is a reference zero axis indicating positions of focal spots of all structures for the frequency $300 \mathrm{GHz}$. For each structure one can distinguish characteristic line segments inclined to this reference axis. The inclination angle is a measure of chromatic aberration. Thus, the smaller the angle is, the smaller the chromatic aberration and the more broadband working of the designed element is assured.

The chromatic aberration diagram was created from energy distributions found in numerical simulations and in experimental evaluation with the same parameters as in a case 

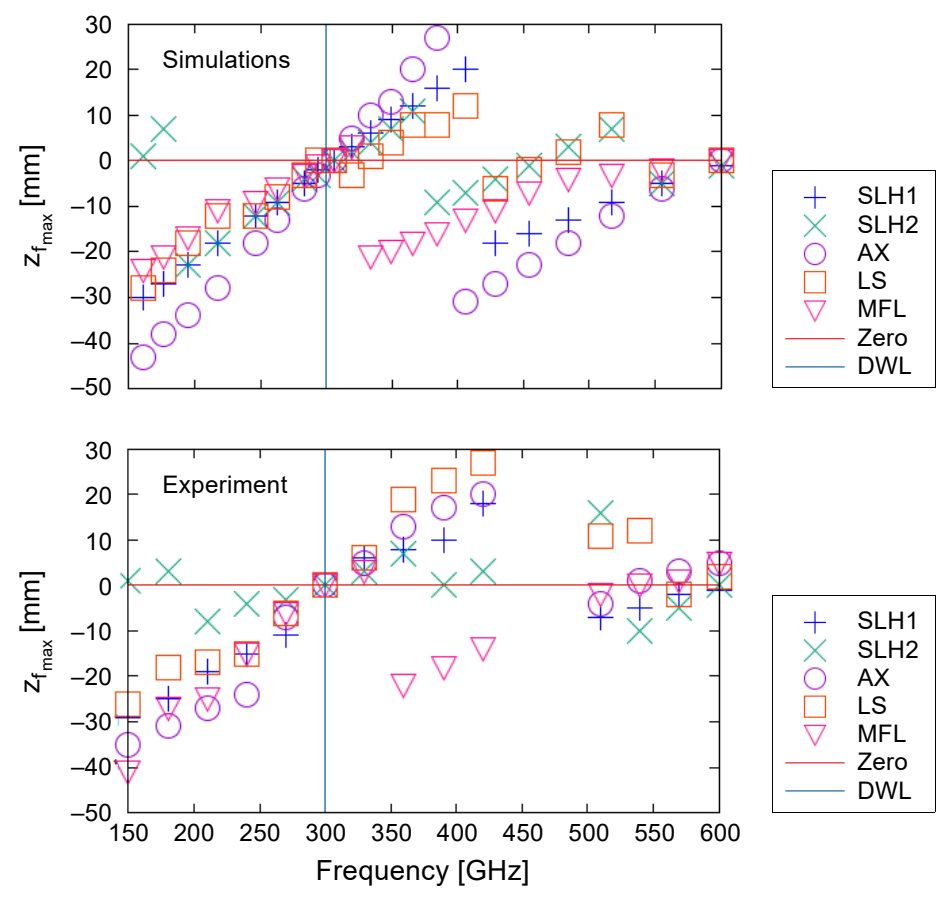

Fig. 2. Diagram of chromatic aberration - the dependence of a focal spot shift on the frequency - computer modelling and experimental results. The red line indicates a reference position of focal spots for a frequency $300 \mathrm{GHz}$ marked with the blue line.

of EnM and PSF distributions. Here, scans along $Z$ axis were performed for different frequencies. Simulations were performed for a wavelength range from 0.5 to $2.02 \mathrm{~mm}$ every $0.04 \mathrm{~mm}$. Distances $z_{\mathrm{d}}$ from $20 \mathrm{~mm}$ to $100 \mathrm{~mm}$ with the scanning step of the detector equal to $1 \mathrm{~mm}$ were similar in computer modelling and in the experiment. In a case of LS element three scans were performed along $Z$ axis for three different $X$ values: $x=0 \mathrm{~mm}$ (along optical axis), $x=-2 \mathrm{~mm}$ and $x=2 \mathrm{~mm}$. The maximal energy of the spot was determined as the maximal value from these three points. The focal spot of the LS element is slightly rotating along $Z$ axis due to the asymmetry of the structure, thus additional scans were necessary to avoid mistakes.

Both simulation and experimental results have the shape of characteristic line-segments oriented at some angle to the zero axis. On both diagrams two lines were marked - one indicating the frequency of $300 \mathrm{GHz}$ (marked with blue line) and second indicating the reference zero axis (red line). Due to the fact that the inclination angle is a measure of chromatic aberration, we tried to find the element having all $z_{\text {max }}$ values as close to zero as possible which provided more broadband working of the designed element. As it was noticed, all segments should intersect the zero axis for the $300 \mathrm{GHz}$ frequency. Kinoforms of the first order intersect zero axis around the 300 and $600 \mathrm{GHz}$, while kinoform of the second order has four intersections: around 150,300, 450 and $600 \mathrm{GHz}$. 
These four segments for SLH2 correspond to four frequency ranges, where structure acts as kinoform of the first, the second, the third and the fourth order.

The axicon (AX) element has the biggest slope relative to the zero axis, but in the 200-250 GHz range a small plateau can be observed. The LS element crosses the zero axis in three points and flattening can be observed around $200-250 \mathrm{GHz}$, then for $300 \mathrm{GHz}$ (noticeable rather in simulation), and for $600 \mathrm{GHz}$. Thus, the LS element has the smallest local slope for these frequency ranges.

\section{Conclusions}

In this paper we analyzed thin focusing structures functioning for broadband illumination at $\mathrm{THz}$ range. Our idea was to compare functioning of optical elements focusing incident radiation in point-like foci or line segments located along the optical axis. We analyzed 5 diffractive structures: two mono-focal lenses (in a form of the first and the second order kinoforms), multi-focal lens, axicon and light sword.

The best achromatic properties were observed for a spherical lens coded as a kinoform of the second order (SLH2), however, it is also two times thicker structure than all other designed (which results in higher attenuation). Thus, we should discuss and compare also the first order kinoforms and in this case a light sword (LS) element seems to give the best frequency coverage. The results from simulations are evident, but experimental data are less satisfactory. It could result from the fact that maximum of energy along optical axis is difficult to observe due to rotational changes of the position of the focal spot.

It can be noticed that axicon (AX) structure has larger focal spots, especially seen in the experiment. This is also the case for the light sword (LS) element, however it should be underlined that the detector size is relatively large and a little bit bigger and more blurred focal spot is not a drawback anymore.

We observe the shift of the focal plane for three structures: multi-focal lens (MFL), light sword element (LS) and axicon (AX). Therefore, our next goal is to optimize starting and ending points of focal line segments to assure not only broadband working but also fixed focal distance, especially for LS and AX.

The best results were obtained for second order kinoform (SLH2), which corresponds to the fact that kinoforms of higher order exhibit achromatic behavior. They are thicker structures than kinoforms of the first order. Thus, taking into account the fact that often the attenuation of material increases with frequency, it can be concluded that when structures are manufactured with a material that has a high absorption coefficient for terahertz radiation, structures like light sword (LS) can be used. They will assure broadband functioning and small attenuation due to small thickness.

Acknowledgements - This study was supported by project 2017/25/N/ST7/02757 from National Science Centre, Poland. The authors would like to thank the Orteh Company for providing LS 6.0 Software used for designing and modelling the diffractive optical elements. 


\section{References}

[1] Jansen C., Wietzke S., Peters O., Scheller M., Vieweg N., Salhi M., Krumbholz N., Jordens C., HochreIn T., Косн M., Terahertz imaging: applications and perspectives, Applied Optics 49(19), 2010, pp. E48-E57, DOI: 10.1364/AO.49.000E48.

[2] JePsen P.U., Cooke D.G., Косн M., Terahertz spectroscopy and imaging - modern techniques and applications, Laser \& Photonics Reviews 5(1), 2011, pp. 124-166, DOI: 10.1002/lpor.201000011.

[3] Zhang X., Zhang Z., Application of terahertz technology in biomolecular analysis and medical diagnosis, [In] Terahertz Spectroscopy - A Cutting Edge Technology, J. Uddin [Ed.], IntechOpen, 2017, pp. 173-190.

[4] Sung S., TAYLOR Z., Quasioptical imaging system design for THz medical imaging application (Conference Presentation), Proceedings of SPIE 9706, 2016, article 970605, DOI: 10.1117/12.2218578.

[5] Shi L., Shumyatsky P., Rodriguez-Contreras A., Alfano R., Terahertz spectroscopy of brain tissue from a mouse model of Alzheimer's disease, Journal of Biomedical Optics 21(1), 2016, article 015014, DOI: 10.1117/1.JBO.21.1.015014.

[6] Humphreys K., Loughran J.P., Gradziel M., Lanigan W., Ward T., Murphy J.A., O’Sullivan C., Medical applications of terahertz imaging: a review of current technology and potential applications in biomedical engineering, [In] The 26th Annual International Conference of the IEEE Engineering in Medicine and Biology Society, IEEE, 2004, pp. 1302-1305, DOI: 10.1109/IEMBS.2004.1403410.

[7] A low cost and fully passive Terahertz inspection system based on nano-technology for security application, PROJECT NUMBER: FP6-NMP-26786; https://cordis.europa.eu/project/rcn/81555/ factsheet/en (accessed July 23, 2019).

[8] Krimi S., Klier J., Jonuscheit J., von Freymann G., Urbansky R., Beigang R., Highly accurate thickness measurement of multi-layered automotive paints using terahertz technology, Applied Physics Letters 109(2), 2016, article 021105, DOI: 10.1063/1.4955407.

[9] Shumyatsky P., Alfano R., Terahertz sources, Journal of Biomedical Optics 16(3), 2011, article 033001, DOI: $10.1117 / 1.3554742$.

[10] Scherger B., Scheller M., Jansen C., Koch M., Wiesauer K., Terahertz lenses made by compression molding of micropowders, Applied Optics 50(15), 2011, pp. 2256-2262, DOI: 10.1364/AO.50.002256.

[11] Bruckner C., Notni G., Tunnermann A., Optimal arrangement of $90^{\circ}$ off-axis parabolic mirrors in THz setups, Optik 121(1), 2010, pp. 113-119, DOI: 10.1016/j.ijleo.2008.05.024.

[12] SiEmion A., Terahertz diffractive optics - smart control over radiation, Journal of Infrared, Millimeter, and Terahertz Waves 40(5), 2019, pp. 477-499, DOI: 10.1007/s10762-019-00581-5.

[13] Marron J.C., Angell D.K., Tai A.M., Higher-order kinoforms, Proceedings of SPIE 1211, 1990, pp. 62-67, DOI: 10.1117/12.17930.

[14] Liebert K., Rachon M., Bomba J., Sobczyk A., Zagrajek P., Sypek M., Suszek J., Siemion A., THz diffractive focusing structures for broadband application, Photonics Letters of Poland 10(3), 2018, pp. 76-78, DOI: $10.4302 /$ plp.v10i3.845.

[15] Rostami A., Rasooli H., Baghban H., Terahertz Technology: Fundamentals and Applications, Springer Science and Business Media, Springer-Verlag Berlin Heidelberg, 2011.

[16] Suszek J., Siemion A. M., Błocki N., Makowski M., Czerwiński A., Bomba J., Kowalczyk A., Ducin I., Kakarenko K., Palka N., Zagrajek P., Kowalski M., Czerwińska E., Jastrzebski C., Świtkowski K., Coutaz J.-L., Kolodziejczyk A., Sypek M., High order kinoforms as a broadband achromatic diffractive optics for terahertz beams, Optics Express 22(3), 2014, pp. 3137-3144, DOI: 10.1364/OE.22.003137.

[17] Soifer V.A., Doskolovich L.L., KaZAnskiY N.L., Multifocal diffractive elements, Optical Engineering 33(11), 1994, pp. 3610-3616, DOI: 10.1117/12.179890.

[18] Kolodziejczyk A., Bara S., Jaroszewicz Z., Sypek M., The light sword optical element-a new diffraction structure with extended depth offocus, Journal of Modern Optics 37(8), 1990, pp. 1283-1286, DOI: 10.1080/09500349014551431. 
[19] Sochacki J., Kolodziejczyk A., Jaroszewicz Z., Bara S., Nonparaxial design of generalized axicons, Applied Optics 31(25), 1992, pp. 5326-5330, DOI: 10.1364/AO.31.005326.

[20] Buralli D.A., Morris G.M., Rogers J.R., Optical performance of holographic kinoforms, Applied Optics 28(5), 1989, pp. 976-983, DOI: 10.1364/AO.28.000976.

[21] Mikula G., Kolodziejczyk A., Makowski M., Prokopowicz C., Sypek M., Diffractive elements for imaging with extended depth of focus, Optical Engineering 44(5), 2005, article 058001, DOI: 10.1117 I 1.1905481.

[22] SyPEK M., Light propagation in the Fresnel region. New numerical approach, Optics Communications 116(1-3), 1995, pp. 43-48, DOI: 10.1016/0030-4018(95)00027-6.

[23] Jaroszewicz Z., Kolodziejczyk A., Sypek M., Gomez-Reino C., Non-paraxial analytical solution for the generation of focal curves, Journal of Modern Optics 43(3), 1996, pp. 617-637, DOI: 10.1080/ 09500349608232770.

[24] Kruth J.P., Wang X., Laoui T., Froyen L., Lasers and materials in selective laser sintering, Assembly Automation 23(4), 2003, pp. 357-371, DOI: 10.1108/01445150310698652.

[25] Scherger B., Wietzke S., Scheller M., Vieweg N., Wichmann M., Koch M., Wiesauer K., Characterization of micro-powders for the fabrication of compression molded THz lenses, Journal of Infrared, Millimeter, and Terahertz Waves 32(7), 2011, pp. 943-951, DOI: $10.1007 / \mathrm{s} 10762-011-9806-5$.

Received July 23, 2019

in revised form August 16, 2019 